\title{
THE PENETRATION OF PARTICULATE MATTER FROM THE CEREBROSPINAL FLUID INTO THE SPINAL GANGLIA, PERIPHERAL NERVES, AND PERIVASCULAR SPACES OF THE CENTRAL NERVOUS SYSTEM
}

\author{
BY \\ J. B. BRIERLEY \\ From the Department of Neuropathology, Institute of Psychiatry, \\ Maudsley Hospital, London
}

The precise termination of the spinal meninges around the emerging nerve roots has been the subject of many investigations since the early work of Quincke (1872) who injected $1 \mathrm{c.cm}$. of a suspension of cinnabar into the lumbar subarachnoid space of dogs. At examination two to four days later it was observed that cinnabar particles had passed on occasion along the lumbar nerves but more frequently along the intercostal.

Key and Retzius (1875) injected various indicators (including gelatin coloured with Berlin blue) into the subarachnoid space under a pressure of $60 \mathrm{~mm}$. of mercury, and noted their progress for some distance along the peripheral nerves. However, in this connexion, Weed (1914) quotes Testut as having pointed out that the pressure employed was responsible for the production of tissue damage, especially in the arachnoid at the site of the emergence of the nerve roots.

Similar findings were recorded by Goldmann (1913) with trypan blue, although the well-known toxic effects of this indicator may detract to some extent from the results obtained.

The classical experiments of Weed (1914) in which an isotonic mixture of potassium ferrocyanide and ferric ammonium citrate was introduced into the cerebrospinal fluid at a pressure only slightly in excess of physiological, did not permit demonstration of prussian blue granules for more than a short distance along the anterior and posterior nerve roots.

Elman (1923) defined the anatomical arrangement of the membranes around the spinal nerve roots using as indicators india ink and the Weed prussian blue mixture. The subarachnoid space was described as terminating in an anamical cul-de-sac at the point where the arachnoid turned inwards to fuse with the pia. No outward passage of ink granules was observed through the membranes in this region, but there was an excretion of the prussian blue solution into arachnoid cell nests lying in close relation to and often embedded in the dura.

A similar failure of india ink particles to pass into the root ganglion or along the nerve was reported by Iwanow and Romodanowsky (1928) in their experiments on dogs, although these workers were the first to describe the outflow of cerebrospinal fluid into lymphatic channels around the nerve roots.

The confinement of thorotrast to the anatomical cul-de-sac of the subarachnoid space was noted by Wustmann (1933) who visualized the indicator by means of $x$ rays. There was, however, a distinct escape of the material into the epidural fat layers.

The behaviour of a fine suspension of india ink (particle size $0.5 \mu$ ) when introduced into the subarachnoid space under physiological pressure conditions was investigated by Brierley and Field (1948) and the outflow of ink from the cul-de-sac into lymphatic channels was described. In this work, only the naked eye appearances in the region of the root ganglia were recorded, the ink granules not appearing to pass beyond the proximal pole of the ganglion and a few millimetres farther along the ventral root.

Somberg (1947) in a review of the literature criticizes many previous workers on the grounds that the indicators employed were either toxic (e.g. trypan blue) or irritant (e.g. cinnabar) and that they were often introduced under abnormally high pressures with possible production of tissue damage as a result.

Hassin (1947) maintained that the subarachnoid space is to be followed in appropriate sections over the surface of the ganglion, the arachnoid "... being 
in close proximity to but not blended or fused with it or the dura mater. That is to say, the subarachnoid space plunges into the spinal ganglion where it becomes continuous with its tissue spaces and finally emerges over the peripheral (post-ganglionic) nerve as its perineural space". Hassin is prepared to accept the experimental evidence of Key and Retzius (1875) and of Funaoka (1930) and his co-workers in which intraneural injection of various dyes was used to demonstrate a communication between the endoneural and perineural spaces of the peripheral nerves and the subarachnoid space. Hassin states that " . . . the remarkable experiments of Funaoka cannot be attributed to faulty technique ", and again " . . the foregoing instances of marvellous experimenting and vast penetration of the injected dyes can hardly be explained by forced pressure ..." ", this latter in spite of an intraneural injection of $25 \mathrm{c.cm}$. of "dekalen ".

The histological pictures presented by Hassin are not convincing evidence of the continuity of the subarachnoid space over the ganglion as is claimed. Such a claim could only be established (within the known limitations of histological technique where tissue spaces are concerned) if truly longitudinal sections of the ganglion and post-ganglionic nerve were presented. The two sections illustrated in Hassin's paper pass obliquely through the ganglion and provide no information as to the disposition of the membranes in the important regions of the middle zone and distal pole.

That the passage of injected material from the peripheral nerve into the subarachnoid space is by no means certain or predictable was shown by Brierley and Field (1949) using as inoculum a volume of $0.05 \mathrm{ml}$. of radioactive phosphorus (as phosphoric acid). It was recorded that an injection carried out in the upper third of the rabbit's sciatic nerve was less likely to enter the cerebrospinal fluid than an injection made at a lower level, but tended to pass directly into the substance of the spinal cord. The use of a larger injection volume may result in more constant entry into the subarachnoid space as a result of some mechanical tissue damage and it may well be doubted if an intraneural injection much in excess of $0.05 \mathrm{ml}$. is a valid indicator of normal anatomical relationships between endoneural and perineural spaces and the subarachnoid space.

The purpose of the present investigation was, first, to define the normal dispositions of the spinal membranes as they pass towards the root ganglion and the ventral nerve root, and secondly to demonstrate the behaviour of a fine sterile particulate indicator introduced into the subarachnoid space under physiological pressure conditions. However, the proved ability of such particles to pass through the arachnoid and dura in certain situations (Brierley and Field, 1948 ; Field and Brierley, 1948) was a reminder that similar regions of membranous permeability might be encountered elsewhere around that part of the subarachnoid space under investigation.

The technique adopted was also expected to provide histological studies of the perivascular spaces of the central nervous system, from which, in view of the survival period of 48 to 72 hours, it would be evident whether or not there was any considerable penetration of these spaces under normal cerebrospinal fluid pressure.

\section{Material and Methods}

Animals.-Eight adult rabbits of weights from 2.0 to $3.0 \mathrm{~kg}$. were used.

Anæsthetic.-The animals were anæsthetized with sodium nembutal $(2.5 \%$ solution intravenously), recovery taking place two to two and a half hours after the operation.

Indicator.-A suspension of india ink in saline, prepared as described previously (Brierley and Field, 1948). Ninety per cent. of the particles have a diameter of $0.5 \mu$, and the range of size is from 0.4 to $1.5 \mu$. The suspension was dialyzed for one hour in a cellophane sac suspended in running tap water and was sterilize immediately before use.

\section{Technique}

The indicator was introduced into the cisterna magna which was approached by a mid-line incision extending $2 \mathrm{~cm}$. above and below the arch of the atlas, the anima lying prone on the table with the head flexed to $90^{\circ}$. The posterior spinal muscles were separated with careful attention to hæmostasis until the margin of the occiput was exposed. The point of attachment of the atlanto-occipital membrane to the bone in the mid-line was defined and the periosteum stripped over the adjacent four or five millimetres of the occipital surface.

The bone was drilled with a fine dental drill (fissure No. 0) at a point in the mid-line $2 \mathrm{~mm}$. above the free posterior margin of the foramen magnum, the drill point being directed obliquely backwards towards the cistern. Perforation of the bone and dura was indicated by the free escape of cerebrospinal fluid into the groove between the posterior spinal muscles. The free fluid was drawn up into a syringe to give a rough check on the volume released. A children's type lumbar puncture needle was swung into place over the hole and slowly introduced until cerebrospinal fluid could be obtained on slight suction. A $2.0 \mathrm{ml}$. syringe filled with india ink suspension was attached to the needle. The indicator ran in rapidly at first, then slowed down and came virtually to rest after the admission of a volume rather less than that of the cerebrospinal fluid withdrawn. In no instance was the pressure of introduction allowed to exceed $120 \mathrm{~mm}$. of the indicator. Immediately on 
withdrawal of the needle the hole was closed with bone wax and the muscles and skin edges apposed with separate layers of silk sutures.

The introduction of the indicator was repeated at 24-hour intervals, an average of three operations being performed on each animal. In this way it was possible to introduce up to $4.5 \mathrm{ml}$. of indicator into one animal without appreciably raising the pressure of the cerebrospinal fluid.

The animals were killed 12 to 24 hours after the last operation by exsanguination through the thoracic aorta under nembutal anæsthesia. A cannula was then tied into the ascending aorta and perfusion carried out with about $1000 \mathrm{ml}$. of physiological saline followed by an equal quantity of $10 \%$ formol saline.

A complete exposure of the spinal cord was obtained by carrying out a laminectomy from sacrum to coccyx. The sciatic nerves were next exposed from the thigh towards the vertebral canal, fine bone forceps being used in the last part of their course to reveal the spinal root ganglia.

\section{Macroscopical Appearances}

Spinal Cord and Nerve Roots.-The spinal cord and meninges presented the typical appearances described in detail elsewhere, the "ink-cuffs" being particularly well developed in all animals of the series. Examination under the dissecting microscope (Fig. 1) showed that the ink had penetrated some distance over the surface of the root ganglion and almost to the distal pole in some cases. This was in marked contrast to the findings previously reported and may be accounted for by the introduction of larger volumes of ink at a site (cisterna magna) more suitable for spread of the indicator to the spinal cord. The distal termination of the "ink-cuff" was noticeably ragged and appeared to consist of more or less parallel black lines of varying thickness. There was a dense deposit of ink in the " axilla" between the nerve roots and the spinal cord.

A proportion of the india ink on the surface of the ganglion lay in the epidural connective tissue, but when this latter had been dissected off the linear disposition of ink particles on the surface of the ganglion still remained.

The ventral nerve roots exhibited only a light speckling of ink particles which died out at a level corresponding to the midpoint of the ganglion, although in some instances there was a thin tail of ink extending distally along the inner edge of the nerve from the tip of the dense " axilla" referred to above.

The extent of the spread of ink over the surface of the root ganglia was by no means uniform throughout the spinal nerves but was constantly maximal in the three large nerve roots contributing to the formation of the sciatic nerve. At higher levels the outward spread of ink diminished, until, in the thoracic region the apex of the "ink-cuff" coincided with the proximal pole of the root ganglion. In the cervical region there was conspicuous migration of ink, largely in the epidural connective tissue and reaching a maximum along the second and third cervical nerves where it was probably responsible for the greater part of the filling of the deep cervical lymph nodes. Removal of the ink-stained epidural tissue revealed that the ink had spread up to and on to the dorsal root ganglion only in the second and third cervical nerves, whereas in the remaining cervical nerves the apex of the "ink-cuff" lay at the proximal pole of the root ganglion.

Central Nervous System.-The distribution of the ink around the circumference of the spinal cord at any one level was seldom uniform. In the cervical region the greater part of the ink lay on the dorsal aspect of the cord and there was little more than a faint speckling on the ventral surface. This unequal distribution was probably due to the dorsal position of the site of introduction. In the thoracic, lumbar, and sacral regions the discrepancy between dorsal and ventral aspects is reduced while the coccygeal cord, ganglia, and roots were invariably surrounded by a dense accumulation of ink which penetrated the dura to stain the epidural connective tissue.

In two animals of the series the bulk of the ink in the spinal subarachnoid space was concentrated in a well-defined strip lying along the dorsal aspect of the cord and extending from the lower end of the cisterna magna to a tapered point at the midthoracic level. In these two animals there was a poor development of the "ink-cuffs" in the lumbar region.

Transverse sections of the spinal cord were examined under the dissecting microscope and showed the presence of a ring of grey haze lying immediately under the pia and most marked in the cervical region on its dorsal aspect. It was impossible to avoid the suggestion that this appearance might be due to the passage of ink from the subarachnoid space through the pia.

The distribution of ink particles around the brain itself was reasonably uniform from case to case and quite similar to that described by other workers (Weed, 1914 ; Hurst, 1932). The greatest accumulation of ink was noted around the medulla, the infundibulum and hypophysis, and over most of the surface of the cerebellum. Dense cuffs of ink had formed around the emerging cranial nerves, particularly the olfactory, optic, and trigeminal. The intensity of the indicator on the surface of the cerebral hemispheres decreased towards the vertex 


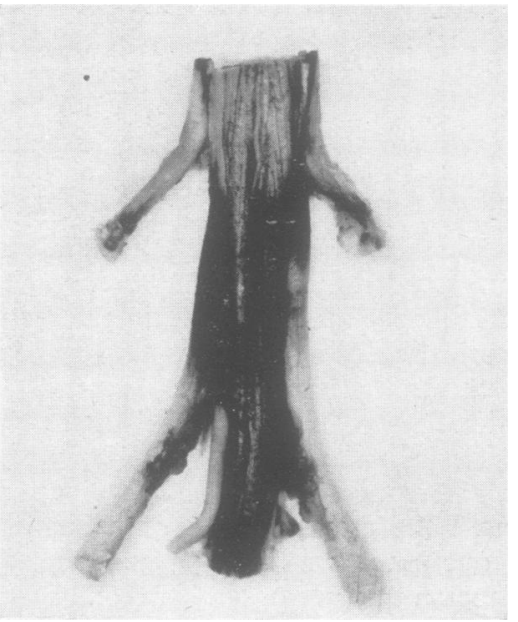

Fig. 1.-Sacral spinal cord of rabbit $(\times 2) 24$ hours after second introduction of india ink. Note spread over surface of root ganglia and dense "axilla" along inner surface of nerves.

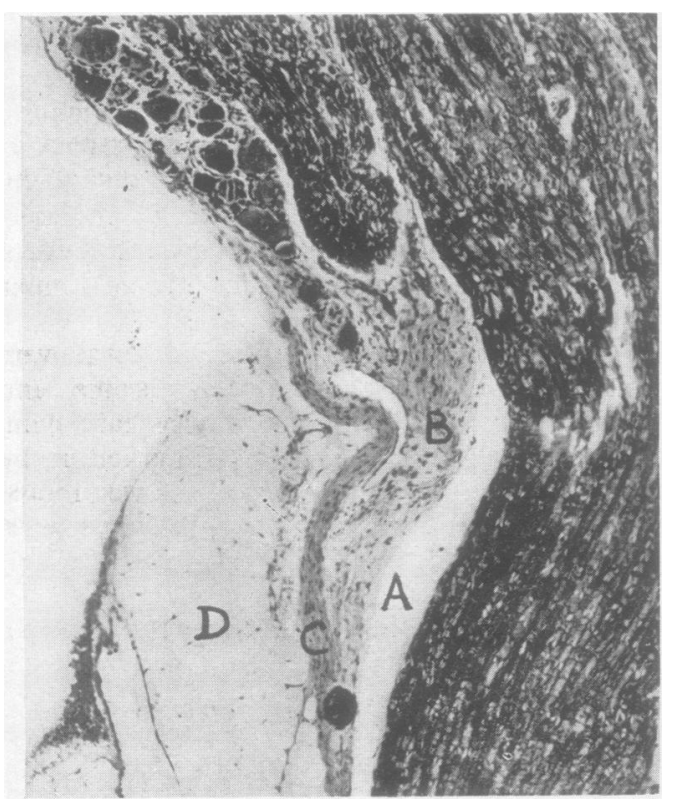

Fig. 3.-Longitudinal section through proximal pole of normal root ganglion $(\times 85)$. A. Subarachnoid cul-de-sac. B. Reduplication of the arachnoid. C. Dura. D. Epidural connective tissue.

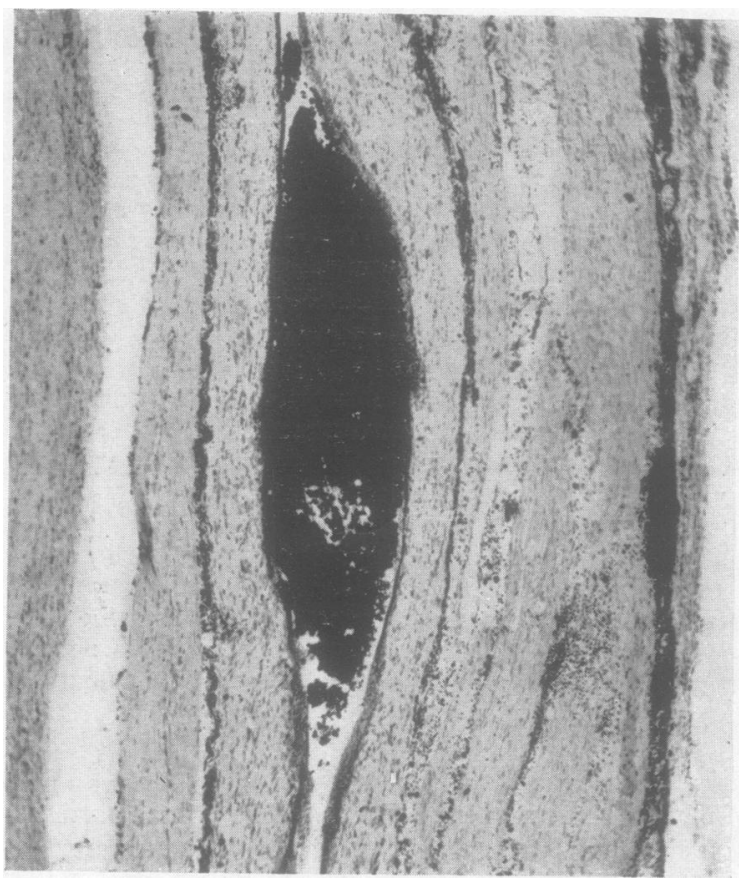

FIG. 2.-Longitudinal section of tip of spinal cord $(\times 60)$ showing dilated termination of central canal containing ink.

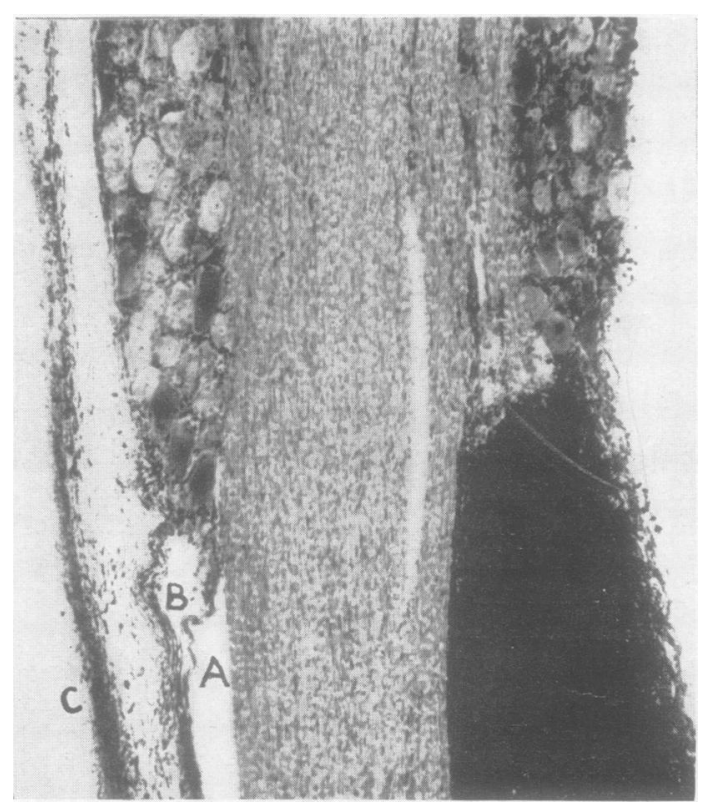

Fig. 4.-Longitudinal section through proximal pole of root ganglion after introduction of ink. $(\times 80)$. Cul-de-sac on right contains dense accumulation of ink and polymorphonuclear cells. ( $\mathrm{K}$ as for Fig. 3.) 
and was in general more intense in relation to the cortical vessels.

Examination of thick coronal slices under the dissecting microscope showed in certain regions the same grey haze beneath the pia as was seen in the spinal cord, but this appearance was confined to certain definite localities. It was constant and most marked in the hypothalamus but was also seen in order of decreasing intensity in the ventral parts of the cerebral cortex, in the cerebral cortex in relation to the choroidal fissure, and throughout the ventral and lateral aspects of the brain stem.

There was a conspicuous absence of ink in the lateral ventricles except for a faint dusting of ink on the choroid plexuses. There was a slight collection of particles in the lowest part of the third ventricle and an increasing amount on passing back through the aqueduct to the fourth ventricle. However, the quantity of ink even in the latter situation was only a fraction of that to be seen in a similar volume of the basal subarachnoid space. This paucity of ink in the ventricular system was in contrast to the situation in the central canal of the spinal cord which appeared as a distinct black dot at all levels of the cord and which terminated in a dilated and ink-filled sac in the lower sacral region (Fig. 2).

\section{Histological Appearances}

Normal Material.-The nerve roots, ganglia, and mixed spinal nerves of several normal animals were examined by means of longitudinal (in the plane of an emerging pair of nerves) and transverse sections. The animals had been perfused with physiological saline under anæsthesia followed at once by $10 \%$ formol saline. The material was embedded in celloidin and cut at 12 to $15 \mu$.

In the material thus prepared, the region of greatest importance lies at the proximal pole of the root ganglion upon which the three spinal membranes converge and where any particulate indicator, intrathecally introduced, can be relied upon to accumulate. Here the question arises, does the subarachnoid space end blindly as a cul-de-sac or is it continued as a narrow or even potential space over the surface of the ganglion? Careful scrutiny of the longitudinal sections has left no doubt, that while the spinal dura passes over the ganglion to form its sheath and ultimately the nerve perineurium, the arachnoid has no such distal extension (Fig. X). Again, the subdural space is easily recognizable for a considerable distance over the ganglion and appears to be continuous with the sub-perineural space of the peripheral nerve, whereas the subarachnoid space becomes narrowed at the proximal pole of the ganglion and terminates in a clearly defined anatomical cul-de-sac. This is the result of the dipping in of the arachnoid membrane towards the margin of the annulus of large cells that forms the proximal pole of the ganglion (Fig. 3). At this point there is a reduplication of the membrane itself and the various layers pass in to blend with the connective tissue of the ganglion. It is to be noted that the apex of the cul-de-sac lies

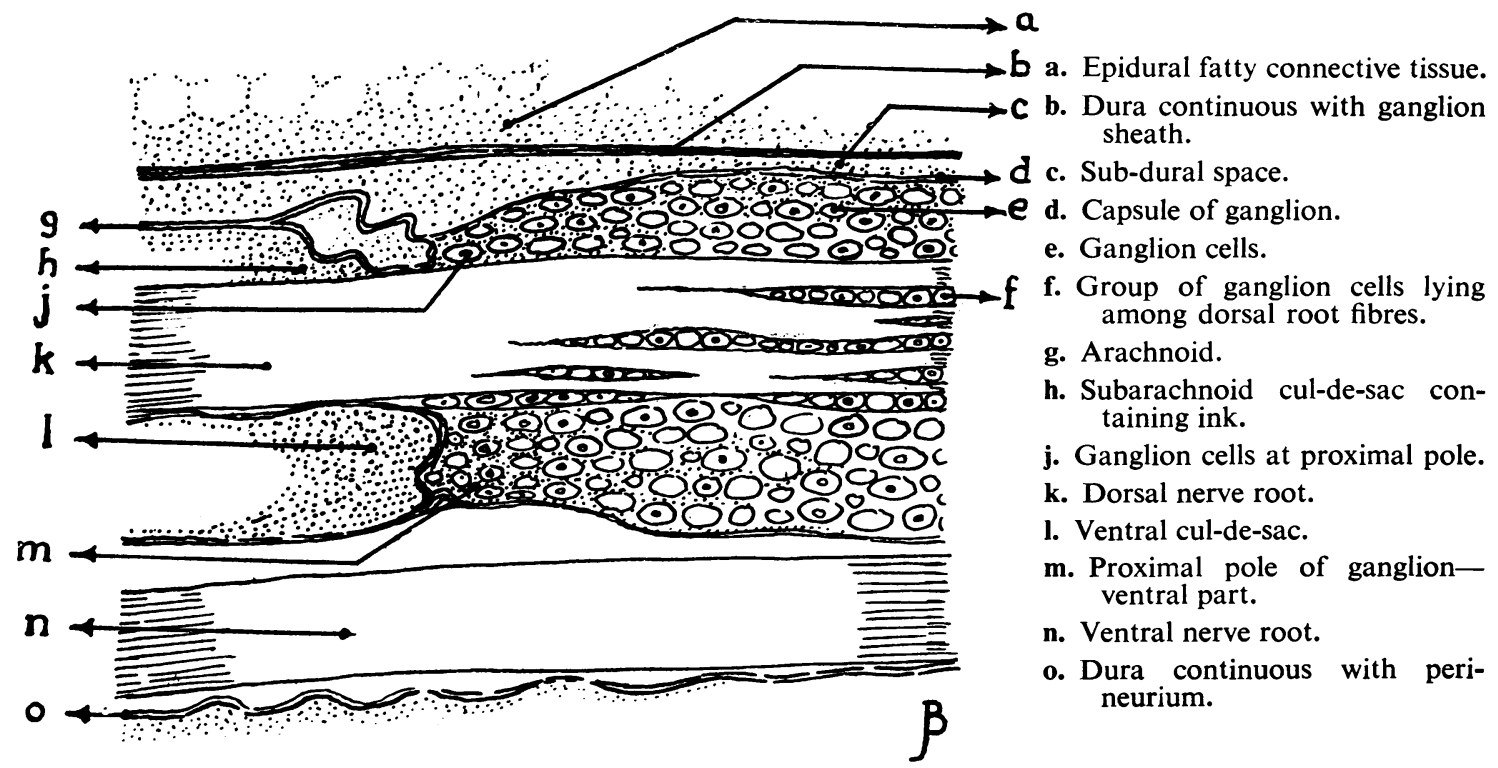

FiG. X.-Diagrammatic longitudinal section through proximal pole of dorsal root ganglion. 
in close relation to the ganglion cells from which it is separated by only a few layers of flattened connective tissue cells (Fig. 4).

Between the dorsal and ventral nerve roots there is a similar cul-de-sac which again terminates in close relation to ganglion cells.

Around the ventral nerve root the subarachnoid space is gradually reduced, the arachnoid apparently fusing with the perineurium and with the superficial connective tissue layers of the nerve at about the mid point of the ganglion.

India Ink.-Within the general subarachnoid space the ink particles tend to accumulate on the deep aspect of the arachnoid rather than on the pia. This distribution is also found in the basal portions of the "ink-cuffs" where there is also a penetration of the arachnoid by the ink which comes to lie in the sub-dural space, along which it penetrates as far as the distal pole of the ganglion (Fig. 6). Extensive permeation of the dura itself is also evident, so that the loose, fatty epidural connective tissue contains a considerable quantity of ink, part of which is destined to enter the terminals of fine lymphatic vessels and so to be conveyed to the general lymph stream.

In the region of the cul-de-sac the disposition of the ink fails to reveal any anatomical continuity of the subarachnoid space over or into the ganglion, there being no narrow wedge or line of particles extending in either of these directions. Nevertheless numerous ink particles are seen to lie among the ganglion cells whose periphery they outline to give a characteristic " wire-netting" appearance (Fig. 5) as briefly reported elsewhere (Field and Brierley, 1948).

A careful study of both transverse and longitudinal sections shows that there are two modes of access of ink particles to the typical peri-cellular position in the ganglion (Fig. Y.) : (a) At the apical zone of the subarachnoid cul-de-sac, ink particles are able to pass through the layers of connective tissue and then to drift slowly around the ganglion cells towards the distal pole of the ganglion. (b) The ink that has penetrated the arachnoid to enter the sub-dural space moves slowly in a distal direction over the

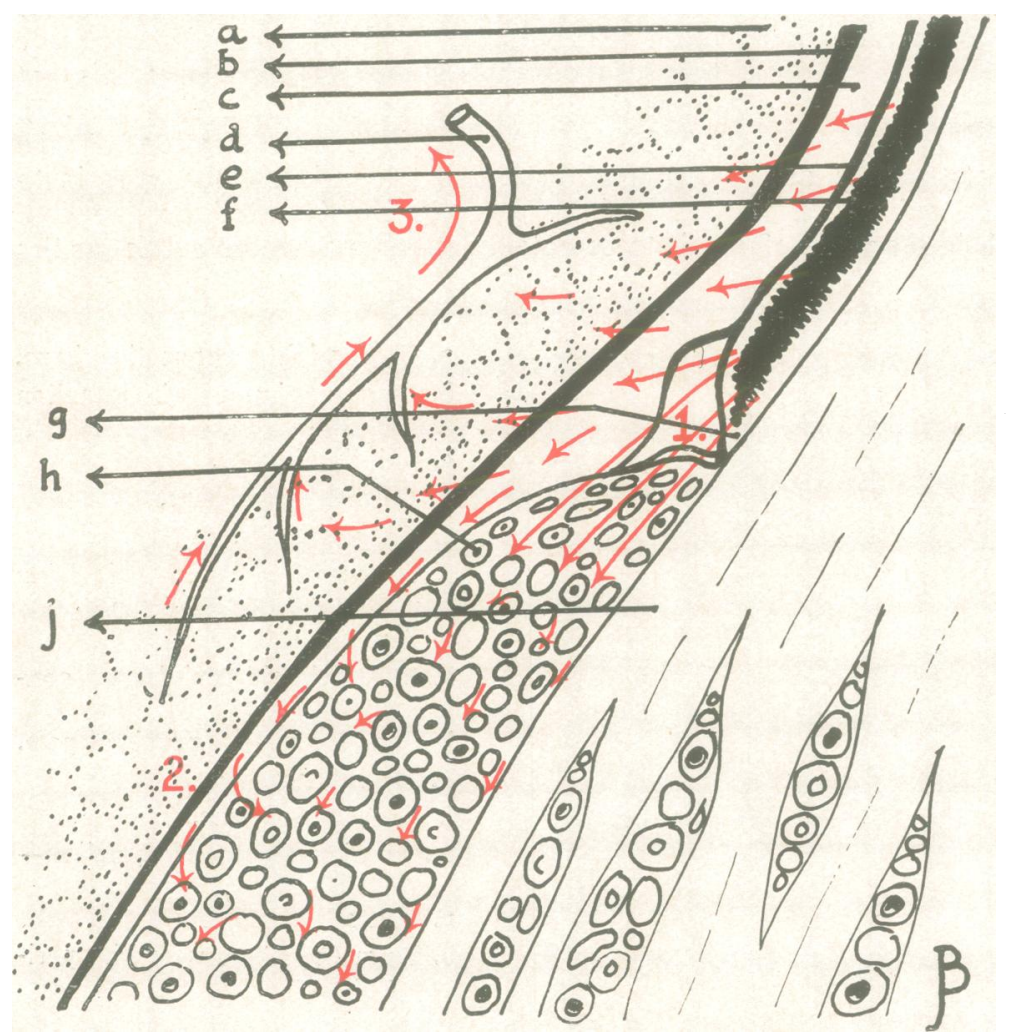

a. Epidural fatty connective tissue.

b. Dura.

c. Sub-dural space.

d. Lymphatic vessel draining epidura tissues.

e. Arachnoid.

f. India ink in subarachnoid space.

g. Subarachnoid cul-de-sac.

h. Ganglion cell.

j. Dorsal nerve root.

1. Outflow into proximal pole of ganglion.

2. Passage back from sub-dural space into peripheral part of root ganglion.

3. Outflow through arachnoid into sub-dural space and thence through dura into lymphatics in epidural connective tissue.

Fig. Y.-Diagram to show escape routes for cerebrospinal fluid from the subarachnoid cul-de-sac. 


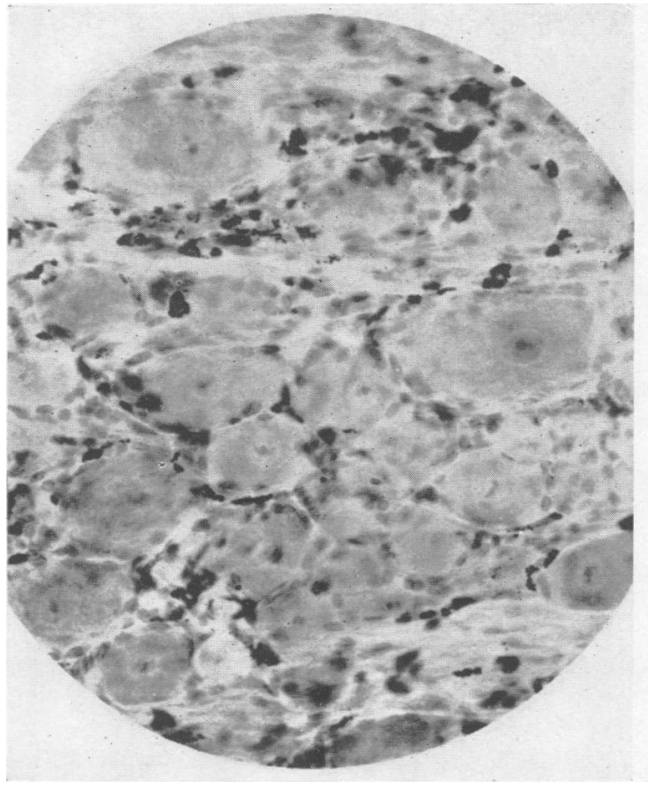

FIG. 5.-Longitudinal section through middle of root ganglion after introduction of ink $(\times 275)$. Note peri-cellular distribution of ink particles.

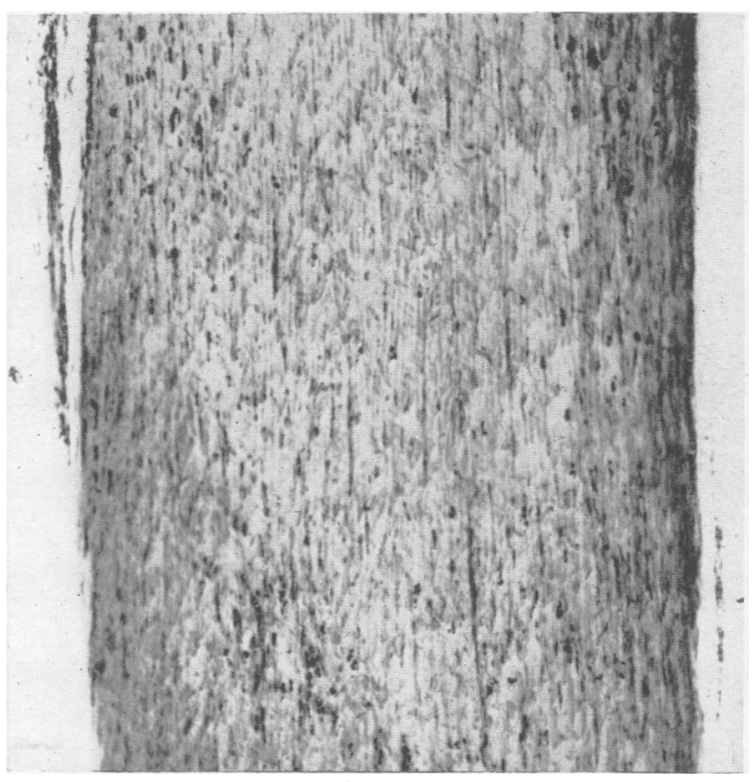

FIg. 7.-Longitudinal section of mixed spinal nerve beyond distal pole of ganglion. ( $\times 75)$. The ink particles lie in parallel columns between the nerve fibres.

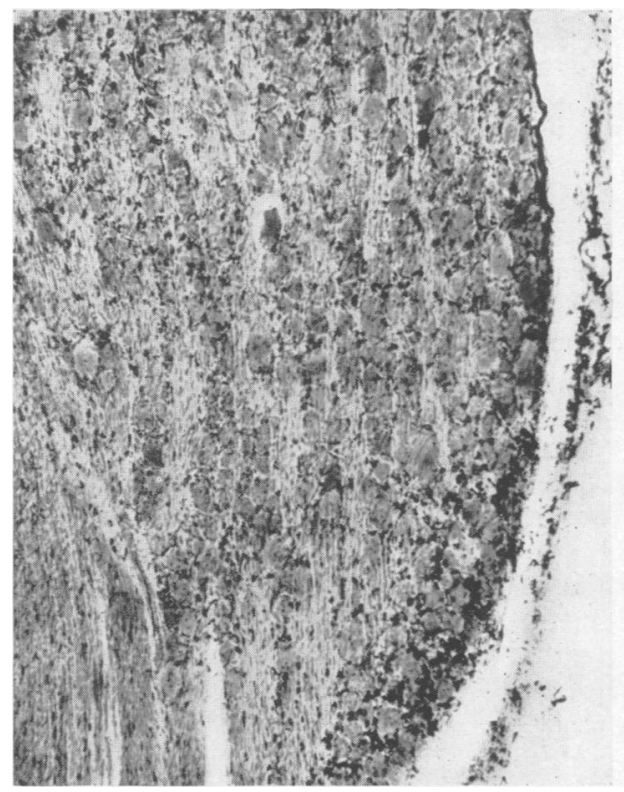

Fig. 6.-Longitudinal section through distal pole of ganglion $(\times 65)$. Note penetration of ink particles from sub-dural space, among the ganglion cells.

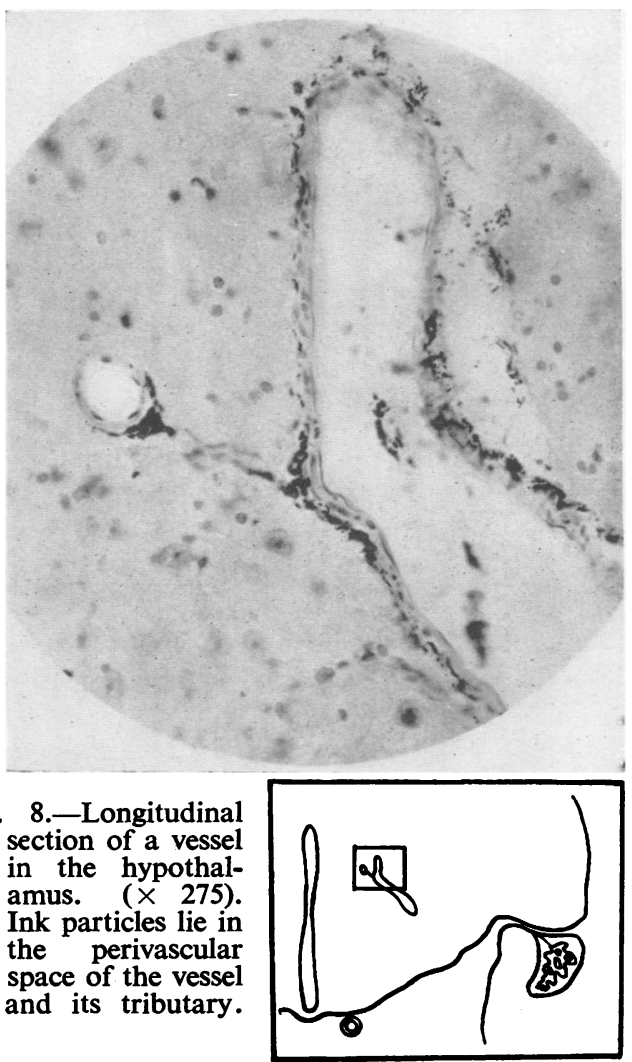


ganglion, and a proportion of the particles pass inwards among the ganglion cells and are again carried towards the distal pole (Fig. 6).

Thus it comes about that in an animal that has received adequate amounts of ink and has survived at least 48 hours there is a distinct capsule of ink particles around almost every cell of the root ganglion, the overall distribution of the ink being fairly uniform.

When the mixed spinal nerve just distal to the ganglion is examined it is seen that the progress of the ink through the ganglion continues into the nerve, where the particles are found lying in parallel columns between the nerve fibres (Fig. 7). The maximum distal spread observed was 4 to $5 \mathrm{~mm}$. from the distal pole of the ganglion. It is emphasized that there is no peripheral concentration of ink in the nerve as would be expected if the subarachnoid space were in direct continuity with the sub-perineural space. The latter space is clearly recognizable beyond the distal pole where it is the continuation of the sub-dural space, yet at this level it is almost devoid of ink, and can therefore contribute little if anything to the total ink visible in the mixed nerve. It must be concluded then that the ink particles lying among the nerve fibres have come from peri-cellular situations in the ganglion substance, as a result of passive transport in a slow centrifugally directed stream.

In the ventral nerve root, the subarachnoid culde-sac, as already pointed out, extends to about the mid-point of the root ganglion and its walls appear to possess the same permeability to ink particles as does its counterpart in the dorsal root. The amount of ink escaping by this route is, however, small, the major centrifugal stream lying in the sub-dural space which is clearly visible in all the sections (Fig. 4 and Fig. 6). Proximal to and alongside the ganglion there is little ink to be seen in the interior of the ventral root, but this amount increases distally, until at the point of junction with the dorsal root the concentration of ink particles in the two neural components is almost equal (Fig. 7). This uniform disposition of ink is then maintained throughout some 4 to $5 \mathrm{~mm}$. of the mixed spinal nerve beyond which it slowly decreases to disappear after a further few millimetres.

Perivascular Spaces of the Central Nervous System.-Serial coronal celloidin sections of the brain and cervical cord were cut and every tenth stained with carmalum, intermediate sections being stained where necessary.

It was at once apparent that a certain proportion of the perivascular spaces in any one section contained ink particles, not only in their mouths, but often to a considerable depth. It was further observed that the perivascular spaces were only well-filled in situations in which the subarachnoid space itself contained obvious accumulations of ink. In general, then, the filling of the spaces reflected the distribution of the indicator over the hemispheres, brain stem, and spinal cord. Thus, in the hypothalamus (Fig. 8) ink-enclosed vessels could be traced from the basal subarachnoid space to within a short distance of the walls of the third ventricle, and in the region of the choroidal fissure of the lateral ventricles numerous perivascular spaces contained ink throughout the whole thickness of the cortex (Fig. 9).

Isolated vessels in the thalamus (Fig. 10) showed limited filling while all degrees of penetration could be seen in cortical vessels, mainly on the ventral and ventro-lateral aspects of the brain (Figs. 11, 12, 13, and 16).

An exceptional quantity of ink around almost every vessel was constantly observed throughout the hippocampal white matter (Fig. 15) lying between the pyramidal cell layer and the fascia dentata.

Similar findings were observed in the globus 0 pallidus the majority of whose vessels were sur-o rounded by small quantities of ink (Fig. 19).

Again, in the cerebellum there was considerable filling of perivascular spaces which could be traced through the Purkinje cell layer into the white core of the folium (Fig. 18).

In the brain stem the depth of penetration of the indicator into the long straight perivascular spaces was more restricted than in the cortical and diencephalic structures and may well be due to the proportionately smaller quantity of ink in the adjacent subarachnoid space. Good penetration was observed at several sites in the medulla (Fig. 14) and repeatedly in the cervical spinal cord (Fig. 17).

The existence of a demonstrable perivascular space was not revealed in any region of the central nervous system except the hippocampus where some unusual shrinkage may well have been responsible for its production. The india ink particles appeared to lie on the outer surface of the vessel in direct contact with the brain substance. The penetration of the ink, although considerable, did not reach the perineuronal spaces, which were again not visible in the celloidin material.

The uniform absence of any perivascular cuffing by inflammatory cells suggested that movement towards the deeper parts of the spaces was not dependent upon a process of phagocytosis. Further, examination under the oil immersion lens showed that most ink particles lay quite free on the vessel adventitia, and the only cells containing ink were sparsely scattered microglia in the nervous tissue 


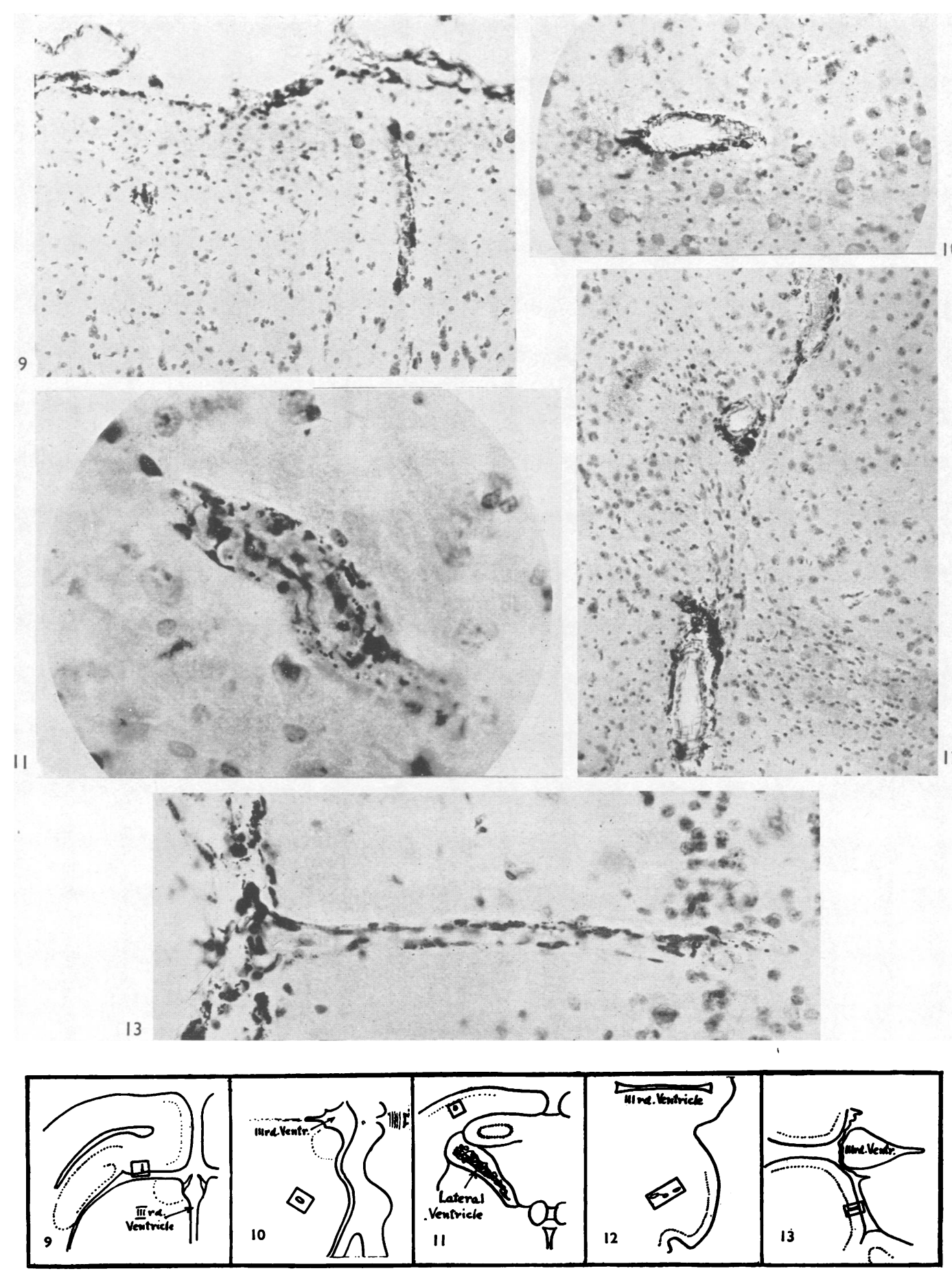

Fig. 9.-Perivascular space containing ink in cortex beside choroidal fissure. $(\times 125$.

FIG. 10.-Vessel of thalamus surrounded by ink particles. $(\times 125$.

FIG. 11.-Small vessel in cortex showing perivascular ink. $(\times 425$.

FIG. 12.-Perivascular ink deposition around sub-cortical vessels in ventral aspect of hemisphere. $(X 125$.

FIG. 13.-Perivascular ink deposition in cortex beside choroidal fissure. $(\times 375$. 


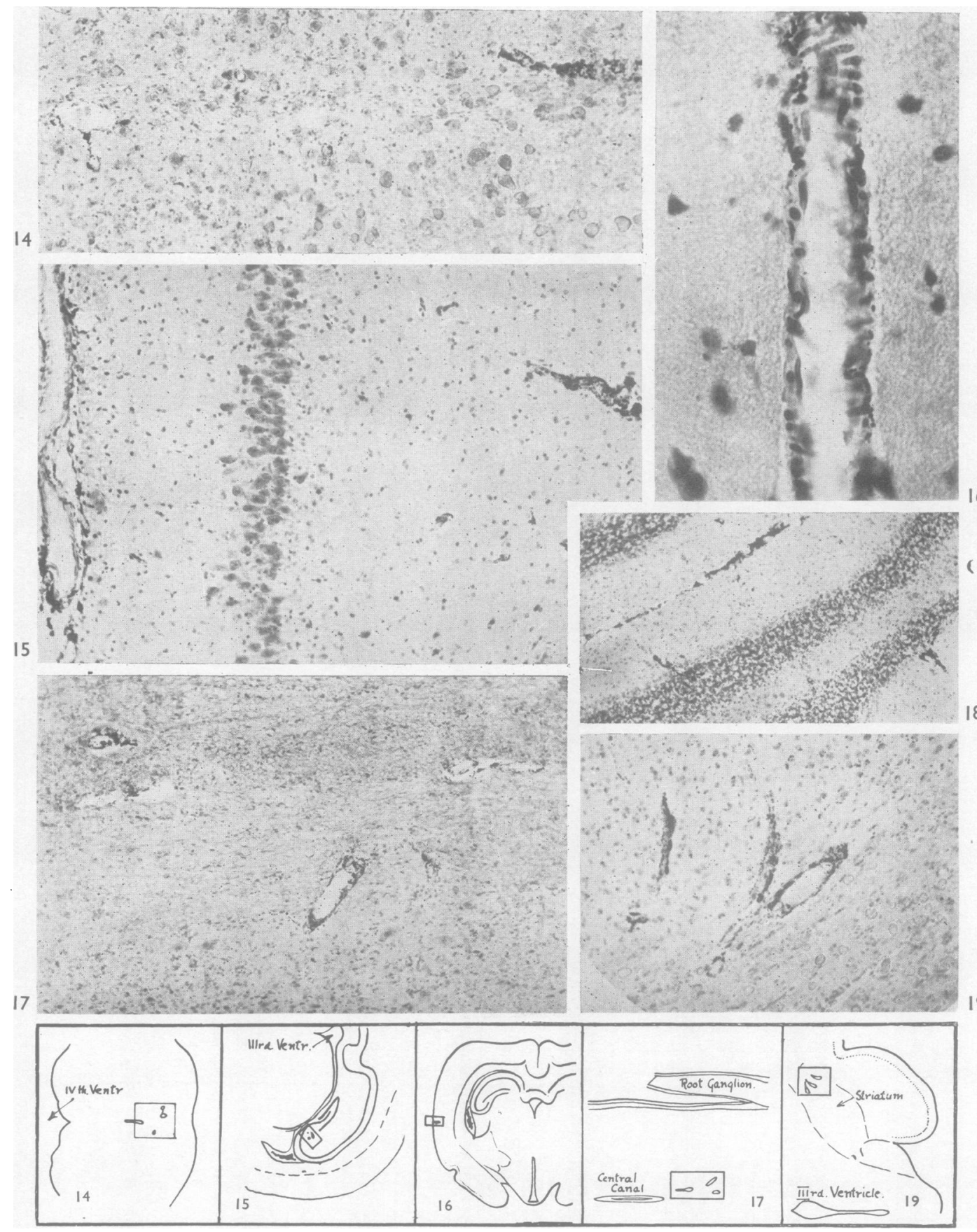

FIG. 14.-Coronal section of lower medulla $(\times 95)$, ventral aspect showing ink deposit around three vessels.

Fig. 15.-Coronal section of Ammon's horn $(\times 110)$, showing subcortical vessel surrounded by ink.

FIG. 16.-Longitudinal section of cortical vessel $(\times 500)$ showing perivascular deposition of fine ink particles and the constriction of the perivascular space at its mouth.

Fig. 17.-Longitudinal section of cervical spinal cord $(\times 90)$ showing ink around vessels in the central grey matter.

FIG. 18.-Cerebellar cortex $(\times 55)$ showing perivascular ink deposits around vessels passing through Purkinje cell layer.

FiG. 19.-Vessels entering globus pallidus $(\times 85)$ surrounded by ink deposits. 
around the perivascular space and in certain regions just below the pia.

\section{Discussion}

The existence of an anatomical cul-de-sac of the subarachnoid space at the proximal pole of the root ganglion and around the ventral root has been established by the present investigation, and confirms the findings of Weed (1914), Iwanow and Romodanowsky (1928), Mulder (1938), and others. No evidence has been found in support of the contention of Hassin (1947) that the subarachnoid space is in anatomical continuity with the tissue spaces of the dorsal root ganglion and the perineural space of the mixed spinal nerve.

Continuity has, however, been demonstrated between the sub-dural space of the nerve roots and the sub-perineural space of the spinal nerve. There is a free passage of particulate matter from subarachnoid to sub-dural space and thence towards the peripheral nerve, a finding which at first glance gives the impression that the subarachnoid space and the sub-perineural space are in direct communication.

From the point of view of physiology and pathology the main interest of the investigation lies in the peculiar permeability of the walls of the subarachnoid cul-de-sac to sufficiently fine particulate matter. From this it is reasonable to conclude that a certain proportion of the cerebrospinal fluid must escape by this route into the ganglion and spinal nerve. However, such an outflow can hardly make any appreciable contribution to the total excretion of the cerebrospinal fluid, and is likely to be of even lesser magnitude than the excretion into lymphatic channels described previously (Brierley and Field, 1948). Nevertheless, this escape of cerebrospinal fluid into the nerve must be taken into account in any consideration of the extent and direction of a possible endoneural fluid stream (frequently and incorrectly referred to as nerve lymph), as it would obviously be difficult to envisage a centripetal fluid flow in any intraneural situation other than within the axis cylinders. That the inter-fibre spaces of the peripheral nerve provide a free and extensive potential pathway for injected material has been demonstrated by Mulder (1938), for a wide variety of indicators, and by Brierley and Field (1949) for radioactive phosphorus and suspensions of india ink. The literature does not as yet provide any convincing evidence of a physiological fluid movement in either a central or a peripheral direction. Mulder (1938) has shown that small intraneural injections of dyes, particulate suspensions, and oily substances are seen to diffuse equally in either direction from the point of injection. It must be concluded then that the centrifugal fluid stream which is responsible for the transport of india ink particles through the dorsal root ganglion to the spinal nerve (in some 48 hours) is probably expended within the first few millimetres of the nerve, and is unable to influence the dispersion of an indicator injected at a more distal site in the nerve.

It is interesting to consider the reasons for the failure of Weed (1914) to note any centrifugal migration of his intrathecally introduced suspension of lamp black. The most probable explanation involves the factors of survival time and the dimensions of the particulate indicator. The duration of Weed's experiments ranged from one to six hours, intervals too brief to permit the slow centrifugal escape of cerebrospinal fluid along the nerve to manifest itself. It has been noted above that some 12 hours are required for the entry of ink particles into the root ganglion, and about 48 hours for the staining of both ganglion and mixed spinal nerve.

Reference has been made elsewhere (Brierley and Field loc. cit.) to the question of particle size and egress from the subarachnoid space. The small size of the particles employed in this work may well facilitate their passage through the wall of the cul-de-sac and the intercellular spaces of the dorsal root ganglion.

The chief pathological problem for which the results of the present investigation may have significance is that of tabes dorsalis whose pathogenesis still lacks adequate explanation. Various hypotheses have been advanced, each suggesting a different anatomical site as the primary point of attack of the spirochæte.

At the present time the dorsal root is usually considered to be the first neural site to be involved in the disease. This structure presents two so-called vulnerable zones : (1) the point of penetration of the spinal pia where the neurilemma is deficient, and (2) the radicular nerve of Nageotte lying just proximal to the root ganglion within the subarachnoid cul-de-sac.

It is tempting to consider the pathogenesis of tabes in the light of the behaviour of india ink particles as described above, providing the initial assumption is made that the spirochætes gain access to the cerebrospinal fluid at an early stage of the disease. It would follow then that spirochætes would accumulate in the cul-de-sac of the spinal nerve roots and particularly in the lumbosacral region. The radicular nerve of Nageotte would then be open to their attacks as well as the ganglion cells in relation to the cul-de-sac. Penetration of the arachnoid (aided by the motility of the organisms) would lead to a permeation of the sub-dural space and the initiation 
of the usual hyperplastic reaction in arachnoid, dura, and epidural tissue.

The Perivascular Spaces.-The somewhat unexpected discovery of particulate matter in the depths of the perivascular spaces at once conflicted with the current conception of an outward movement of fluid from the perineuronal spaces to the subarachnoid space, as advanced by Weed (1914), Schaltenbrand and Bailey (1927), and Kubie (1928). In the absence of passive transport by phagocytes there is at present no explanation for this unusual finding. The ink suspension, teing isotonic with the cerebrospinal fluid, cannot be held responsible for the generation of an abnormal fluid flow. Further, the maintenance of the animal in a normal state of hydration would not set up the reversed flow normally associated with the intravenous injection of hypertonic solutions.

There is a possibility that the experimental technique for the introduction of the indicator may be in part responsible. The free escape of cerebrospinal fluid from the drill hole effects a partial emptying of the subarachnoid space and it may well be that some drainage of the perivascular spaces occurs at the same time. The subsequent inflow of the ink refills the subarachnoid space, and the restoration of normal cerebrospinal fluid pressure and volume may temporarily reverse the "normal" flow within the perivascular spaces to permit some ingress of particles. It seems unlikely, however, that the latter could be at all extensive in view of the fact that abnormal (low) pressure conditions exist for only a minute or so during each introduction. If there is a resumption of an outward fluid streaming it is to be expected that some washing out of the particles would take place to leave a minimal deposit in the depths of the spaces. The histological appearances suggest that this is not the case, as in many instances the deposition of the ink is uniform throughout the perivascular space.

It may be concluded, then, that under physiological pressure conditions fine particulate suspensions in the cerebrospinal fluid are capable of penetrating the perivascular spaces in a period of 24 to 48 hours and that the extent of this penetration is related to the quantity of the indicator in the subarachnoid space. It is probable that with adequate and uniform filling of the whole space there would be a considerable degree of filling of most of the perivascular spaces of the central nervous system.

The remarkable constancy with which ink particles were found in the perivascular spaces of the hippocampus and globus pallidus recalled the involvement of these regions in the anoxia resulting from such agents as carbon monoxide, potassium cyanide, ether, morphine, respiratory failure, and dinitrobenzol poisoning (reviewed by Meyer, 1937). In addition siderophilic deposits occur normally in these two zones in man and inorganic iron is frequently demonstrable in and around the walls of the vessels of the globus pallidus. Alexander (1942) has also pointed out that the two most vulnerable structures of the human brain, the globus pallidus and the cornu Ammonis, are both supplied by the anterior choroidal artery (in man).

The presence of perivascular spaces readily accessible to cerebrospinal fluid may be yet another aspect of the unusual vascular relations of these parts.

Reference has already been made elsewhere (Field and Brierley, 1948) to a possible relationship between the distribution of the lesions of poliomyelitis and the penetration of the central nervous system by virus conveyed along perivascular spaces. The results described above have revealed a far more extensive invasion of perivascular spaces than was previously envisaged, and may well raise anew the role of the cerebrospinal fluid in the pathogenesis of the disease.

Summary

The literature relating to the subarachnoid "cul-de-sac" is reviewed.

A technique for introducing an india ink suspension into the cisterna magna of the rabbit without resultant rise in intracranial pressure is described.

Forty-eight hours after the introduction typical "ink-cuffs" are seen at the proximal poles of the root ganglia.

There is naked eye evidence of spread of the ink over the surface of the ganglia, particularly in the ganglia of the sciatic nerve.

The distribution of ink over the cerebral hemispheres, brain stem, and cerebellum is described.

Histological examination revealed (normal material) that the subarachnoid space terminates near the proximal pole of the root ganglion as a well defined cul-de-sac.

India ink particles accumulate in the cul-de-sac whence they pass into the sub-dural space, into the substance of the ganglion, and finally into the mixed spinal nerve.

The perivascular spaces of the central nervous system contain amounts of india ink that vary with the degree of filling of the adjacent subarachnoid space.

The perivascular spaces of the hippocampus, globus pallidus, and cerebellum were well filled in all members of the series. 
The physiological and pathological significance of these findings is discussed.

The author records with pleasure his indebtedness to Professor A. Meyer for his valuable criticism, to Mrs. E. Beck for the photomicrographs, to Mrs. H. Cox for the histological preparations, and to Mr. P. Glenn for skilful assistance in the operative procedures.

\section{REFERENCES}

Alexander, L. (1942). Res. Publ. Ass. nerv. ment. Dis., 21, 77.

Brierley, J. B., and Field, E. J. (1948). J. Anat., Lond., 82, 153.

- (1949). Journal of Neurology, Neurosurgery and Psychiatry, 12, 86

Elman, R. (1923). Bull. Johns Hopk. Hosp., 34, 99.

Field, E. J., and Brierley, J. B. (1948), J. Anat., Lond., 82, 198.

Funaoka, S. (1930). "Eine Injektionsmethode des Nervensystems," Arbeiten aus der dritten Abteilung des anatomischen Institutes der Kaiserlichen Universität Kyoto. Series A. 1.

Goldmann, E. E. (1913). “Vitalfärbung am Zentralnervensystem. Beitrag zur Physio-Pathologie des Plexus chorioideus und der Hirnhäute." Berlin.

Hassin, G. B. (1947). J. Neuropath. exp. Neurol., 6, 172.

Hurst, E. W. (1932). J. Path. Bact., 35, 41.

Iwanow, G., and Romodanowsky, K. (1928). Z. ges. exp. Med., 58, 596.

Key, A., and Retzius, G. (1875). "Studien in der Anatomie des Nervensystems und des Bindegewebes." Stockholm.

Kubie, L. S. (1928). Brain, 51, 244.

Meyer, A. (1937). Proc. R. Soc. Med., 29, 1175. (Section of psychiatry, p. 49.)

Mulder, J. D. (1938). Acta. neerl. Morphol., 1, 289.

Quincke, H. (1872). Arch. Anat. Physiol., Lpz. 153. Schaltenbrand, G., and Bailey, P. (1927). J. Psychol. Neurol., Lpz., 35, 199.

Somberg, H. M. (1947). J. Neuropath. exp. Neurol., 6, 166.

Weed, L. H. (1914). J. med. Res., 31, 93.

Wustmann, O. (1933). Dtsch. Z. Chir., 238, 529.

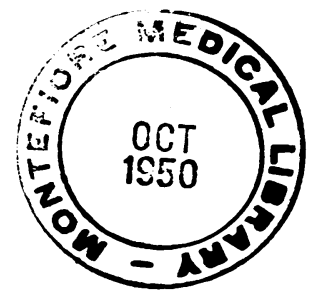

